\title{
The Pharmacological Activity of the Wenjing Decoction in Recurrent Spontaneous Abortion
}

\author{
Linhui Cao, ${ }^{1}$ Hui Chen, ${ }^{2}$ Yanxia Huang, ${ }^{1}$ Liudan Chen, ${ }^{1}$ Mengru Kang, \\ and Junxiong Liang ${ }^{1}$ \\ ${ }^{1}$ Department of Traditional Chinese Medicine, Sun Yat-sen Memorial Hospital, SunYat-sen University, Guangzhou 510120, \\ Guangdong, China \\ ${ }^{2}$ Department of Obstetrics and Gynecology, Sun Yat-sen Memorial Hospital, Sun Yat-sen University, Guangzhou 510120, \\ Guangdong, China
}

Correspondence should be addressed to Junxiong Liang; ljunx@mail.sysu.edu.cn

Received 8 September 2020; Revised 18 March 2021; Accepted 1 April 2021; Published 14 April 2021

Academic Editor: Wei Hsiung Yang

Copyright (c) 2021 Linhui Cao et al. This is an open access article distributed under the Creative Commons Attribution License, which permits unrestricted use, distribution, and reproduction in any medium, provided the original work is properly cited.

Background. Recurrent spontaneous abortion (RSA) is intractable infertility and can be ameliorated with the use of traditional Chinese medicine preparation, the Wenjing decoction. This study aimed to identify the therapeutic mechanism of Wenjing decoction on specific target proteins involved in RSA. Methods. Wenjing decoction contains Wuzhuyu, Danggui, Chuanxiong, Guizhi, Shengjiang, Banxia, Gancao, Ejiao, Mudanpi, Chishao, Dangshen, and Maidong. Using TCMSP and BATMAN databases, we queried for active ingredients and predicted their target proteins by BATMAN. Using the edgeR package, we analyzed the differentially expressed genes (DEGs) in the GSE121950 database between control samples and RSA $(n=3)$. The interaction between DEGs and the predicted target proteins was identified by the Venn diagram. Using the Cytoscape software and clusterProfiler package, enrichment analysis was conducted for the intersected target proteins. Additionally, the protein-protein interaction (PPI) network and pharmacological network were generated using the Cytoscape software. Results. In total, 31, 2, 7, 7, $5,13,93,11,29$, and 21 active ingredients were identified from Wuzhuyu, Danggui, Chuanxiong, Guizhi, Shengjiang, Banxia, Gancao, Mudanpi, Chishao, and Dangshen, respectively. Additionally, 100 intersected target proteins were revealed by the Venn diagram. Moreover, 98 functional terms and 24 pathways (including C-type lectin receptor signaling pathway, chemokine signaling pathway, leukocyte transendothelial migration, fluid shear stress, and atherosclerosis, and AGE-RAGE signaling pathway in diabetic complications) were enriched. In the PPI network, 10 proteins involved in these five pathways were identified, namely, TNF- $\alpha$ (tumor necrosis factor- $\alpha$ ), IL-10 (interleukin-10), TLR4 (Toll-like receptor 4), JUN (Jun proto-oncogene), IL-1B (interleukin-1-beta), CYBB (cytochrome b558 heavy chain gene), PTGS2 (prostaglandin-endoperoxide synthase 2), APOE (apolipoprotein E), SPI1 (salmonella pathogenicity island 1), and MPO (myeloperoxidase) which showed higher degrees. Conclusion. The abovementioned genes and pathways might be involved in the pharmacological activity of Wenjing decoction in RSA.

\section{Introduction}

Abortion involves pregnancy termination before 28 weeks, with fetal weight below $1000 \mathrm{~g}$ [1]. Abortion can be classified as spontaneous or induced abortion, and recurrent spontaneous abortion (RSA) involves two or more consecutive spontaneous abortions [2]. Abortion manifests as postmenopausal vaginal bleeding and abdominal pain, although some women do not exhibit any clinical symptoms [3].
Nearly $50 \%$ of women with RSA have identifiable etiologies, such as chromosomal abnormalities, cervical insufficiency, immune dysfunction, maternal endocrine abnormalities, maternal reproductive tract abnormalities, reproductive tract infection, and thrombotic susceptibility $[4,5]$. RSA is intractable infertility clinically and poses a global therapeutic challenge, accounting for $1-5 \%$ of total pregnancies [6]. Therefore, it is vital to understand RSA pathogenesis to improve therapy. 
Some traditional Chinese medicines (TCMs) have shown promise in women with recurrent miscarriage by improving embryo development and live birth rate [7]. The TCM Wenjing decoction improves blood circulation and eliminates stasis and has been used for alleviating gynecological diseases, including amenorrhea, dysmenorrhea, and paramenia [8]. Wenjing decoction could significantly alleviate pain in women with primary dysmenorrhea and could be used for primary dysmenorrhea treatment [9]. The modified Wenjing decoction repairs abnormal ovarian function in gynecological cold coagulation blood stasis by reducing ovarian oxidative damage [10]. Wenjing decoction is involved in vascular remodeling, microvascular contraction, and microcirculation and helps restore the normal ovarian function in cold coagulation blood stasis syndrome [11]. Therefore, Wenjing decoction alleviates RSA by warming the channel, dispelling cold, nourishing blood, and removing blood stasis. However, the mechanisms underlying Wenjing decoction activity in RSA have not been identified.

TCM typically has multiple components and multiple targets. The active ingredients and candidate targets can be explored by network pharmacology $[12,13]$. In this study, the Wenjing decoction was evaluated by active ingredient screening, target protein prediction, cross-validation, enrichment analysis, and protein-protein interaction (PPI) network and pharmacological network construction. Our findings might help understand the pharmacological mechanisms involved in the Wenjing decoction effect in RSA.

\section{Materials and Methods}

2.1. Composition of TCM Preparation. The TCM preparation of the Wenjing decoction contained Wuzhuyu (Tetradium ruticarpum) $15 \mathrm{~g}$, Danggui (Angelica sinensis) $10 \mathrm{~g}$, Chuanxiong (Ligusticum chuanxiong hort) $10 \mathrm{~g}$, Guizhi (Cinnamomum cassia Presl) $10 \mathrm{~g}$, Shengjiang (Zingiber officinale Roscoe) $10 \mathrm{~g}$, Banxia (Pinellia ternata) $20 \mathrm{~g}$, Gancao (Glycyrrhiza uralensis Fisch) $10 \mathrm{~g}$, Ejiao (Colla corii asini) $10 \mathrm{~g}$, Mudanpi (Paeonia suffruticosa) $10 \mathrm{~g}$, Chishao (Radix Paeoniae Rubra) $10 \mathrm{~g}$, Dangshen (Codonopsis pilosula) $10 \mathrm{~g}$, and Maidong (Ophiopogon japonicus) $30 \mathrm{~g}$. Based on TCM names, the medicinal ingredient information (including molecular weight (MW), molecule name, lipid-water partition coefficient $(\mathrm{A} \log \mathrm{P})$, the number of constituents, the number of hydrogen bond donors/acceptors (Hdon/Hacc), blood-brain barrier (BBB), intestinal epithelial permeability (Caco-2), drug-likeness (DL), oral bioavailability (OB), and drug half-life (HL)) was extracted from the Traditional Chinese Medicine Systems Pharmacology (TCMSP, https:// tcmspw.com/index.php) database [14].

2.2. Screening of TCM Active Ingredients. The active ingredients were identified using the (ADME) parameters [15] from the TCMSP database. ADME studies evaluate the absorption, distribution, metabolism, and elimination of exogenous chemicals by the body and included DL, HL, and OB. This analysis used conventional screening parameters for drug composition. The cutoff criteria were DL $\geq 0.18$ and $\mathrm{OB} \geq 30 \%$.
2.3. Target Protein Prediction for the Active Ingredients. Using the BATMAN (http://bionet.ncpsb.org/batman-tcm/) [16] and TCMSP [14] databases, the target protein prediction for the active ingredients was performed. The BATMAN database first selected the target proteins from the DrugBank, Therapeutic Target Database (TTD), and Kyoto Encyclopedia of Genes and Genomes (KEGG) databases and ranked the predicted targets in descending order. The target proteins were selected for each small molecule drug candidate using a threshold of a score of $>20$. The BATMAN database was used to predict the differentiating drug-target interactions using binary classification for target protein prediction of active ingredients. The classification feature eigenvalue of the predicted drug-protein interaction was considered as the maximum eigenvalue of the similarity scores between the predicted drug-protein interaction and all known interactions.

\subsection{Cross-Validation of the Target Proteins of the Active} Ingredients. The raw data from the GSE121950 was downloaded from the Gene Expression Omnibus (GEO, http://www.ncbi.nlm.nih.gov/geo/) database. This dataset was deposited by Huang et al. [17] and included RNA-seq data from decidua tissues on three RSA samples and three control samples. The original reads were filtered as follows: the reads with sequencing adaptor were removed, and the reads with $\mathrm{N}$-base number exceeding one were filtered out. Subsequently, the reads with low-quality $(Q \leq 20)$ bases exceeding $40 \%$ of the total bases were deleted, and the reads with length $<35$ were omitted. Using the Tophat Software (v2.1.0, http://ccb.jhu.edu/software/tophat, the default parameters) [18], the clean reads were mapped to the human reference genome (GRCh38). Based on featureCounts software (v1.6.0, http://subread.sourceforge.net) [19], read count information for the alignment of each gene was obtained following the human gene annotation information provided by GENCODE (Release 25, https://www. gencodegenes.org/) [20]. The reads with the annotation information of "protein_coding" were taken as mRNAs, and the reads with the annotation information of "antisense," "sense_intronic," "lincRNA," "sense_overlapping," "processed_transcript," "3 prime_overlapping_ncrna," and "non_coding" were considered as lncRNAs.

Using the edgeR package (version 3.4, http://www. bioconductor.org/packages/release/bioc/html/edgeR.html)

[21], the raw counts were standardized and converted to log2 counts per million $(\log C P M)$ values. The differential expression analysis for RSA vs. control groups was conducted. All mRNAs/lncRNAs were analyzed to obtain corresponding $p$ values and log fold change (FC) values. The mRNAs/lncRNAs with $p$ values of $<0.05$ and $|\operatorname{logFC}|>1$ were considered as differentially expressed genes (DEGs). The target protein intersection of the active ingredients and DEGs was analyzed subsequently.

2.5. Enrichment Analysis of the Intersected Target Proteins. The Gene Ontology (GO)_biological process (BP) enrichment analysis of the intersected target proteins was 
conducted using the ClueGO + CluePedia plug-in Cytoscape software (http://www.cytoscape.org) [22]. The adjusted $p$ value $\leq 0.01$ was the threshold for GO_BP enrichment analysis. In addition, the $\mathrm{R}$ package clusterProfiler [23] was used for the pathway enrichment analysis of the intersected target proteins, and a $p$ value $\leq 0.01$ was set as the threshold.

2.6. PPI Network for the Intersected Target Proteins and Pharmacological Network Construction. The interactions among the intersected target proteins were predicted by the STRING database (http://string-db.org/) [24], and the PPI network was built using the Cytoscape software [22]. The required confidence (combined score) $>0.4$ was the cutoff criterion for the PPI selection. In addition, the pharmacological network involving the TCM-active ingredient-target proteinpathway relationships was built by the Cytoscape software [22].

\section{Results}

3.1. Composition of the TCM Preparations and Active Ingredient Screening. The TCM data in the TCMSP database were queried with the keywords to obtain the chemical composition and other relevant information for each TCM. The data showed $176,125,189,220,265,116,280,55,119$, 134, 55, and 3 constituents in Wuzhuyu, Danggui, Chuanxiong, Guizhi, Shengjiang, Banxia, Gancao, Mudanpi, Chishao, Dangshen, Maidong, and Ejiao, respectively. The constituents were screened further to identify ingredients with the best ADME parameters. There were 31, 2, 7, 7, 5, 13, $93,11,29$, and 21 active ingredients in Wuzhuyu, Danggui, Chuanxiong, Guizhi, Shengjiang, Banxia, Gancao, Mudanpi, Chishao, and Dangshen, respectively (Table 1).

3.2. Target Protein Prediction and Cross-Validation. The BATMAN online tool was used to predict the target proteins for the active ingredients in the TCMSP database, and the target records for 10 herbs, including Wuzhuyu, Danggui, Chuanxiong, Guizhi, Shengjiang, Banxia, Gancao, Mudanpi, Chishao, and Dangshen, were obtained. In total, we identified 933 target proteins of 49 active ingredients from the 10 TCMs after screening the targets with a score larger than 20 in BATMAN.

From the raw data of GSE121950, a total of 17163 mRNAs were annotated. Differential expression analysis showed that there were 1371 DEGs (including 453 upregulated and 918 downregulated genes) between RSA and the control samples (Figure 1). The Venn diagram showed the presence of 100 intersecting target proteins among the 933 target proteins of the active ingredients and the 1371 DEGs (Figure 2 and Supplementary Table 1).

3.3. Enrichment Analysis of the Intersected Target Proteins. For the 100 intersected target proteins, 98 GO_BP terms were enriched significantly. The pie diagram showed the involvement of 23 different functions, such as "cellular chemotaxis" (13.27\%), "reactive oxygen species biosynthetic process" (7.55\%), "regulation of nitric oxide biosynthesis" (7.35\%), "positive regulation of the vasculature development" (7.35\%), "regulation of inflammatory response" (6.12\%), and others (Figure 3). In addition, 24 KEGG pathways were enriched for the 100 intersected target proteins. The enriched pathways included primarily "AGE-RAGE signaling pathway in diabetic complications," “TNF signaling pathway," "osteoclast differentiation," "C-type lectin receptor signaling pathway," "chemokine signaling pathway," "leukocyte transendothelial migration," and "MAPK signaling pathway"(Figure 4).

3.4. PPI Network for the Intersected Target Proteins and Pharmacological Network Construction. In the PPI network for the intersected target proteins, 84 nodes and 365 edges existed (Figure 5). In particular, the top 10 nodes, including tumor necrosis factor- $\alpha$ (TNF- $\alpha$ ), interleukin-10 (IL-10), Toll-like receptor 4 (TLR4), c-Jun (JUN), interleukin- $1 \beta$ (IL$1 B)$, cytochrome b558 heavy chain gene (CYBB), prostaglandin-endoperoxide synthase 2 (PTGS2), apolipoprotein E (APOE), Salmonella pathogenicity island 1 (SPI1), and myeloperoxidase (MPO), from the PPI network were screened (Table 2). Combined with the TCM-active ingredient-target protein-pathway relationships, a pharmacological network, involving 10 TCMs, 49 active ingredients, 100 target proteins, and 24 pathways, was constructed (Figure 6).

\section{Discussion}

In this study, we screened $31,2,7,7,5,13,93,11,29$, and 21 distinct active ingredients from the TCM preparations of Wuzhuyu, Danggui, Chuanxiong, Guizhi, Shengjiang, Banxia, Gancao, Mudanpi, Chishao, and Dangshen. In total, 933 target proteins for the active ingredients in the TCMSP database were predicted and 1371 DEGs between RSA and control samples were identified from GSE121950. Venn diagram showed 100 intersected target proteins between the 933 target proteins of the active ingredients and 1371 DEGs. Among the 100 intersected target proteins, 98 GO_BP terms and 24 pathways were enriched significantly.

The PPI network of the intersected target proteins, TNF$\alpha$, IL-10, TLR4, JUN, IL-1B, CYBB, PTGS2, APOE, SPI1, and MPO, showed higher degrees. The etiologies of RSA have been extensively identified previously, including chromosomal abnormalities, cervical insufficiency, immune dysfunction, maternal endocrine abnormalities, maternal reproductive tract abnormalities, reproductive tract infection, and thrombotic susceptibility $[4,5]$. TNF- $\alpha$ overexpression in the decidual tissues and peripheral blood of RSA patients was detected, showing correlation with disease occurrence, and might serve as a candidate indicator for RSA $[25,26]$. The $I L$ 10 levels were significantly lower in RSA than during normal pregnancy, and decreased $I L-10$ levels could be correlated to pathologic pregnancies, while increased $I L-10$ to healthy pregnancies [27]. The involvement of TLR4 and C-C chemokine ligand 2 (CCL2) in RSA development was shown 
TABle 1: The constituents and active ingredients in the traditional Chinese medicines.

\begin{tabular}{lccc}
\hline Medicine & Before screening (number) & After screening (number) & Source \\
\hline Wuzhuyu & 176 & 31 & TCMSP \\
Danggui & 125 & 2 & TCMSP \\
Chuanxiong & 189 & 7 & TCMSP \\
Guizhi & 220 & 5 & TCMSP \\
Shengjiang & 265 & 13 & TCMSP \\
Banxia & 116 & 93 & TCMSP \\
Gancao & 280 & 11 & TCMSP \\
Mudanpi & 55 & 29 & TCMSP \\
Chishao & 119 & 21 & TCMSP \\
Dangshen & 134 & 0 & TCMSP \\
Ejiao & 3 & 0 & TCMID \\
Maidong & 55 & 219 & TCMID \\
Total (including the repeats) & 1679 & & \\
\hline
\end{tabular}

TCMSP, Traditional Chinese Medicine Systems Pharmacology; TCMID, Traditional Chinese Medicine Integrative Database.

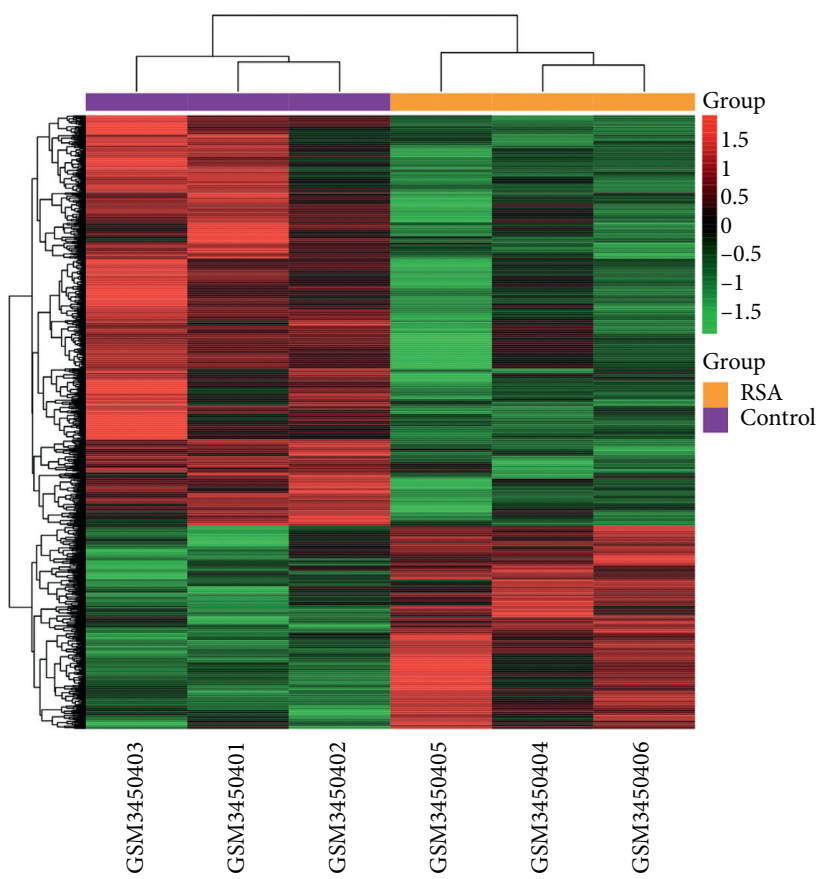

(a)

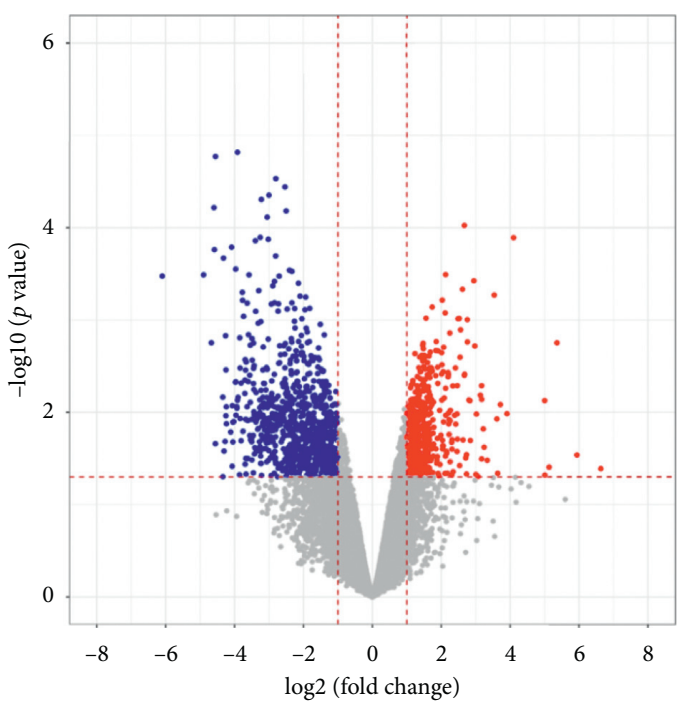

- Upregulated

- Downregulated

(b)

FIGURE 1: The differential expression analysis of GSE121950. (a) Heatmap displayed the differentially expressed genes (DEGs). Red and green represent up- and downregulation, respectively. Orange and purple represent recurrent spontaneous abortion (RSA) and control groups, respectively. (b) Volcano plot displayed the DEGs. Red and blue dots represent up- and downregulated genes, respectively.

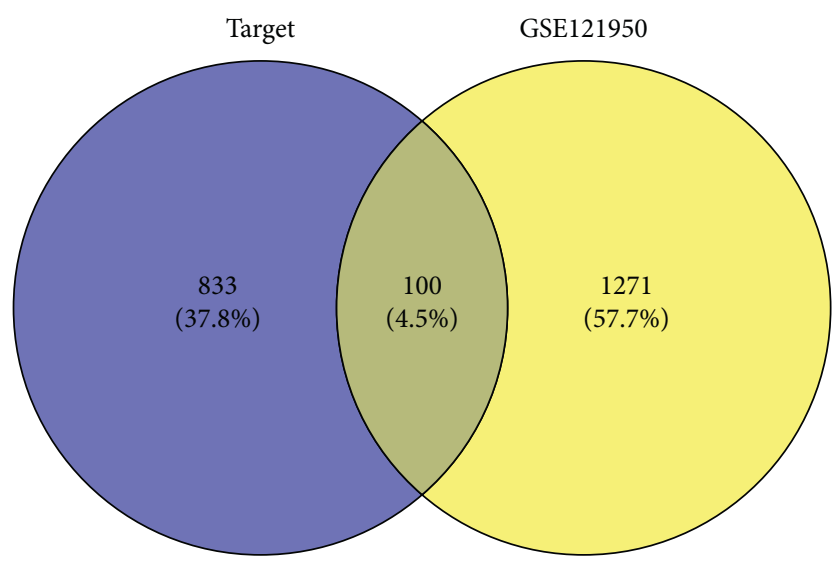

FIGURE 2: The Venn diagram of the intersected target proteins among the 933 target proteins and 1371 DEGs in GSE121950. 


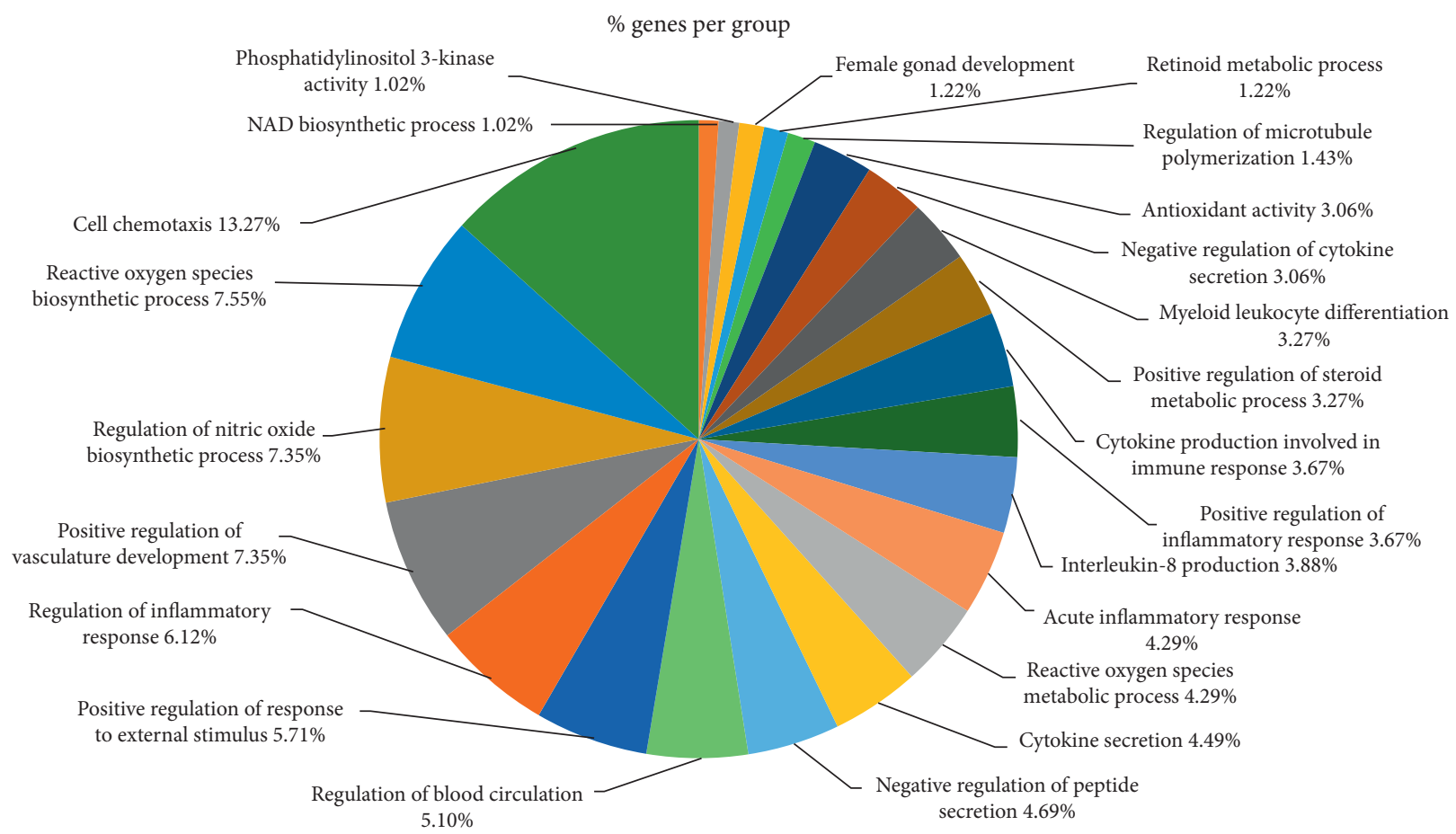

FIGURE 3: The pie chart showing the proportion of Gene Ontology (GO) terms involving the intersected target proteins.

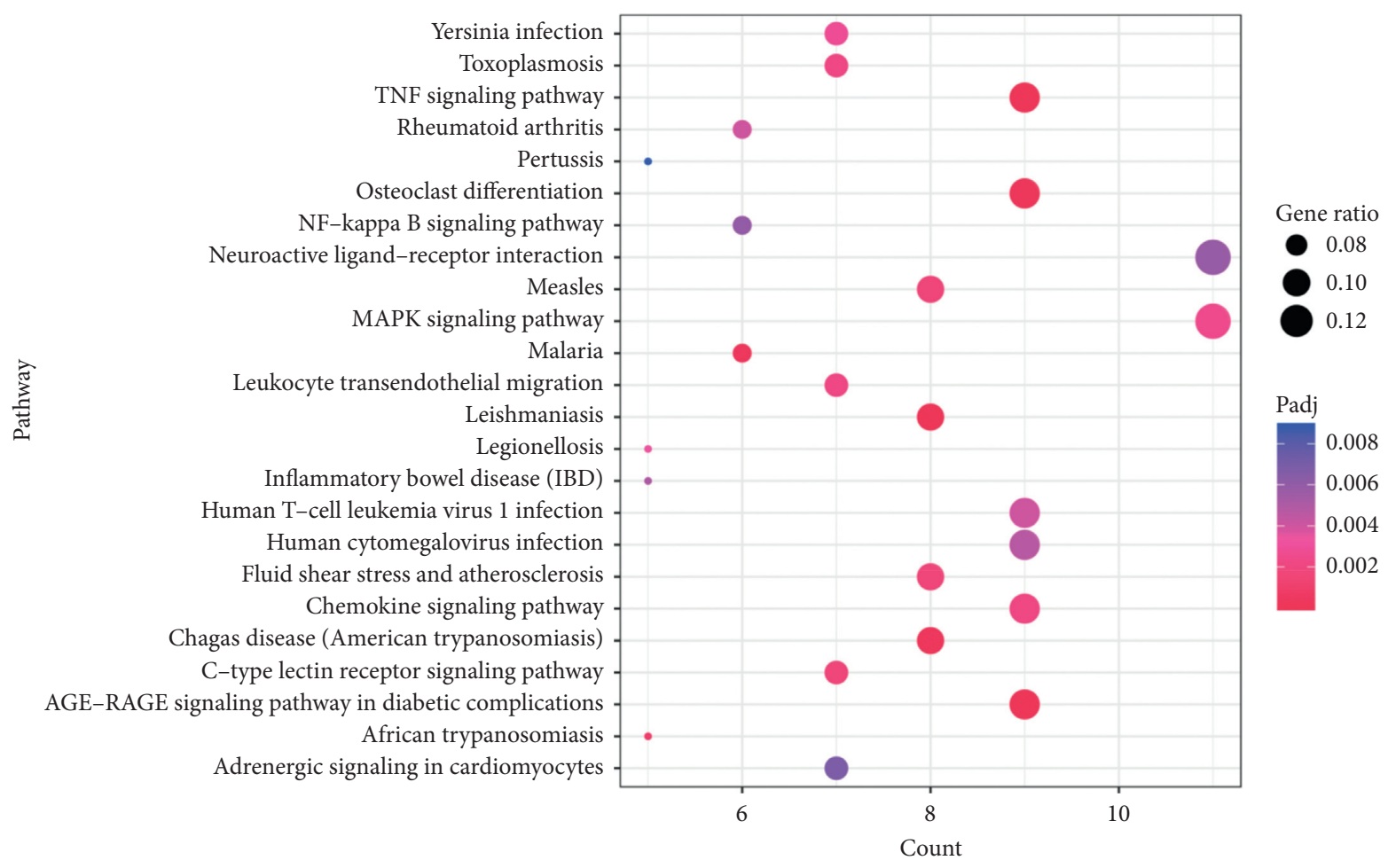

FIgURE 4: The bubble diagram exhibiting the pathways for the intersected target proteins. The vertical axis represents the pathway name, while the horizontal axis shows the enrichment gene number. The larger the ratio of enriched genes to the total genes, the larger the dot size is. A brighter red dot indicates a smaller $p$ value.

before, and the serum TLR4 and CCL2 levels in pregnant women could serve as RSA indicators [28]. Reduced TLR4 and increased TLR2 induced by ligand treatment of spermatozoa influenced unexplained RSA [29]. Therefore, in RSA,
TNF- $\alpha, I L-10$, and TLR4 might be targets for Wenjing decoction.

The $I L-1 B$ (C-511T) polymorphism may promote the development of recurrent miscarriages, while the $I L-6$ 


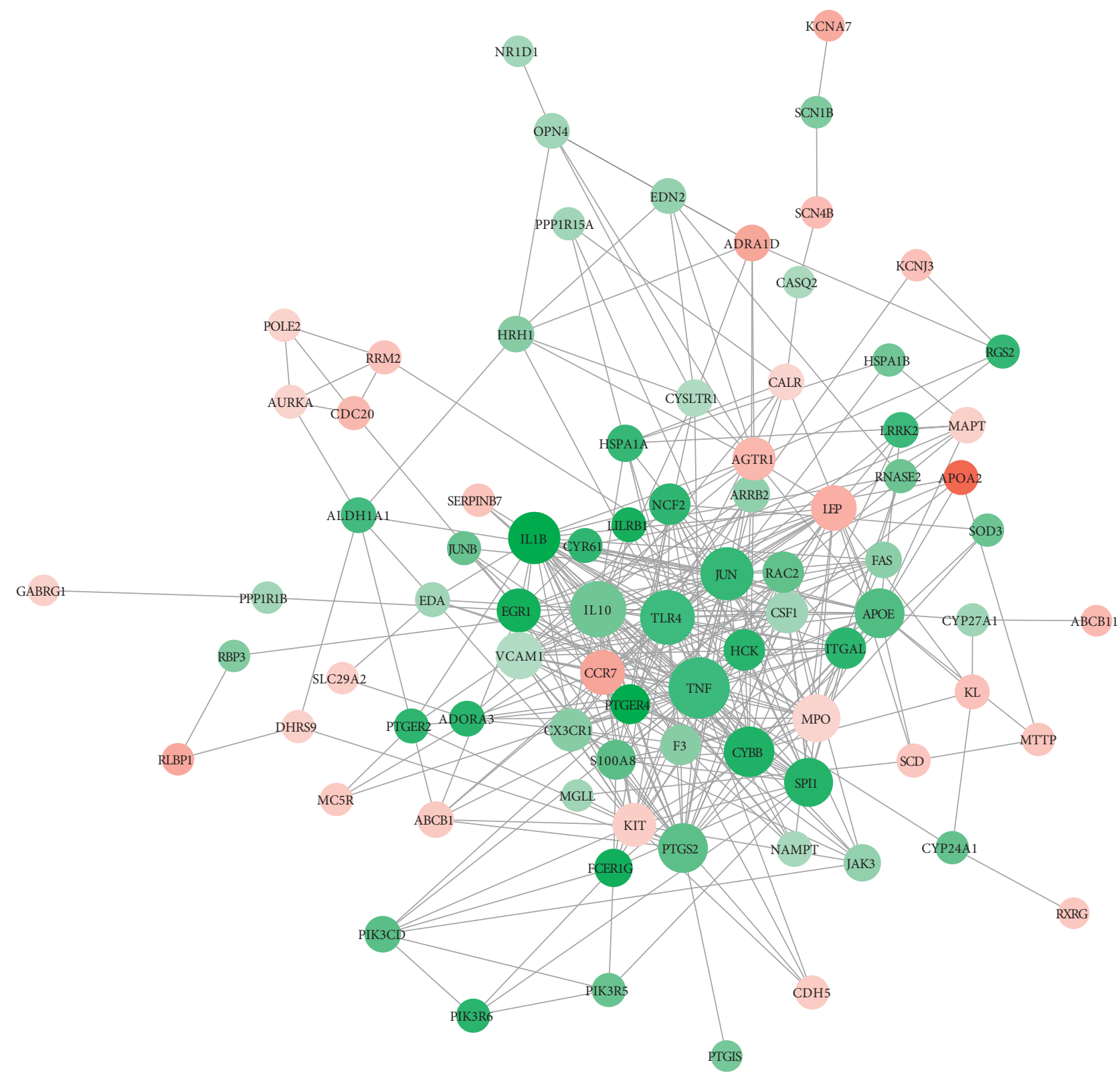

FIgURE 5: The protein-protein interaction (PPI) network for the intersected target proteins. The stronger the connection, the larger the dot is. Red and green represent up- and downregulation, respectively. Darker color indicates greater fold change.

TABLE 2: The top 10 nodes in the protein-protein interaction network.

\begin{tabular}{lccc}
\hline Gene & Degree & Betweenness & Closeness \\
\hline TNF- $\alpha$ & 37.0 & 1138.1646 & 0.58450705 \\
IL-10 & 31.0 & 767.9433 & 0.557047 \\
TLR4 & 29.0 & 448.88934 & 0.5354839 \\
JUN & 27.0 & 832.55804 & 0.5092025 \\
IL-1B & 26.0 & 255.20244 & 0.5253165 \\
CYBB & 24.0 & 324.6068 & 0.51552796 \\
PTGS2 & 23.0 & 619.34546 & 0.5092025 \\
APOE & 23.0 & 805.83795 & 0.5092025 \\
SPI1 & 22.0 & 391.1927 & 0.47428572 \\
MPO & 21.0 & 217.95062 & 0.48255813 \\
\hline
\end{tabular}




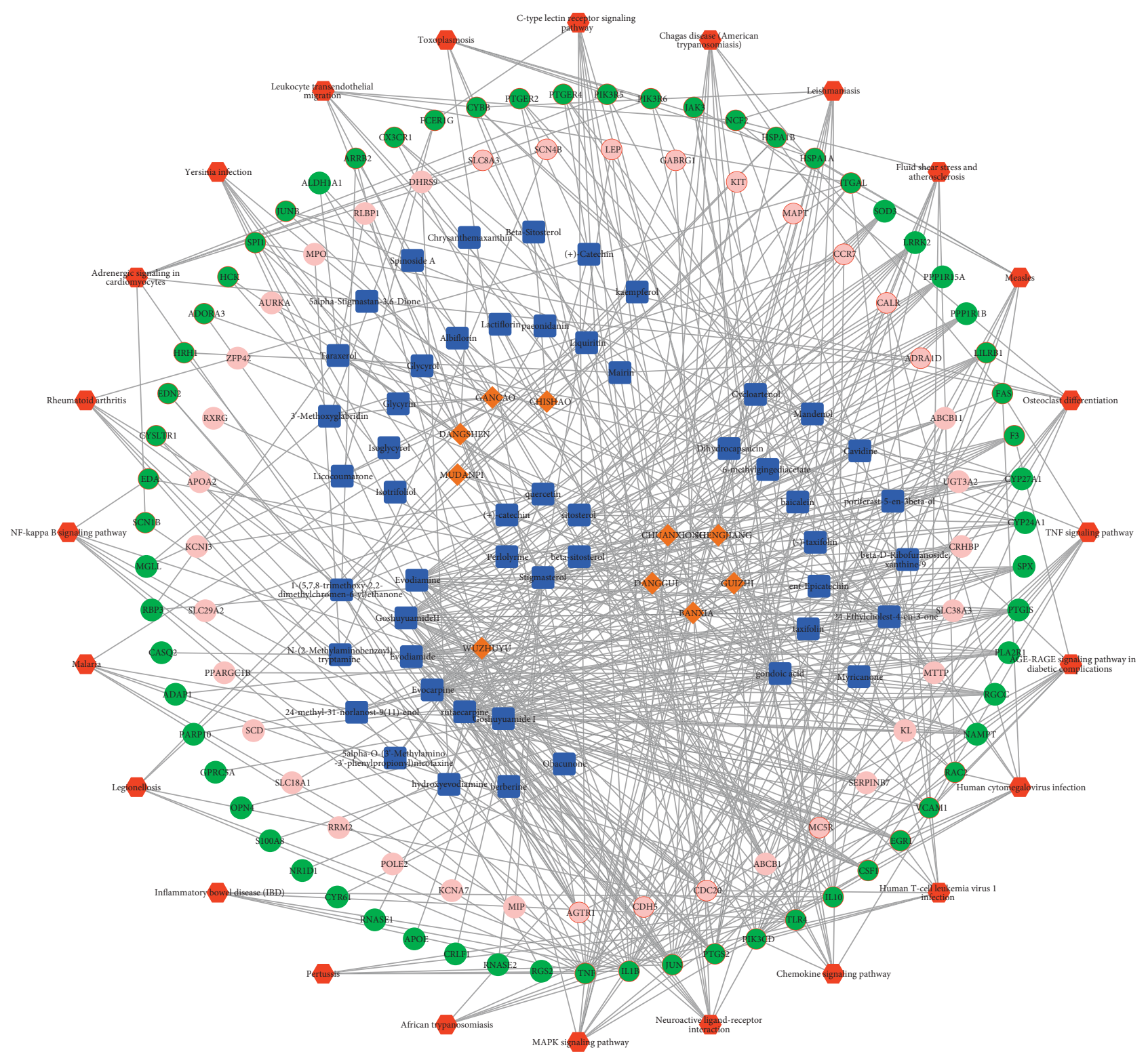

FIgURE 6: The pharmacological network. Brown diamond, blue square, red and green circles, circles with a red border, and red hexagon represent TCMs, active ingredients, target proteins, pathway-associated target proteins, and pathways, respectively.

(G-634C) polymorphism may reduce its risk [30]. DangguiHonghua by modulating 42 targets, including PTGS2, might play a pharmacological role in treating blood stasis syndrome [31]. Apo E3/4, E4/4 genotypes are more frequent in women with recurrent pregnancy loss (RPL) compared to control women, and these genotypes might cause RPL by increasing the thrombophilic risk factors [32]. The levels of the oxidative stress biomarkers, including $M P O$, are higher in women with RPL exhibiting metabolic syndrome, and the oxidative stress, subclinical inflammatory state, and metabolic syndrome contribute to pregnancy outcome prediction in women with RPL [33], thus suggesting that in RSA, $I L-1 B$, PTGS2, APOE, and MPO might also be targeted by Wenjing decoction.

Enrichment analysis of the 100 intersected target proteins showed enrichment of "AGE-RAGE signaling pathway in diabetic complications," "TNF signaling pathway," "osteoclast differentiation," "C-type lectin receptor signaling pathway," "chemokine signaling pathway," "leukocyte transendothelial migration," and "MAPK signaling pathway." A previous study showed the contribution of the C-type lectin receptors in tailoring the adaptive responses and innate recognition, and several C-type lectin receptors function in protective antifungal immunity [34]. Systemic immune responses and abnormal maternal local have shown correlations with RSA; especially, antiphospholipid antibodies and maternal-fetal immune tolerance disturbance affect both autoimmune RSA and alloimmune RSA [35, 36]. Chemokines are a family of chemoattractant proteins, which can trigger immune and inflammatory responses, cell polarization, and prevention of HIV-1 infection [37]. The migration of 
leukocytes through activated venular walls is a basic immune response, which is necessary for the entry of effector cells into the inflamed tissues [38]. Investigations on the role of AGEs and the receptor of AGEs (RAGE) in vascular disease and blood pressure showed the importance of the AGE-RAGE axis in vascular disease, micro- and macrocirculation [39]. Thus, these pathways could be involved in the pharmacological activity of the Wenjing decoction against RSA. TNF- $\alpha$, IL-10, TLR4, JUN, IL-1B, CYBB, PTGS2, APOE, SPI1, and MPO were involved in the pharmacological network pathways and might be responsible for the pharmacological activity of Wenjing decoction.

This study is a preliminary research to screen the primary targets of Wenjing decoction. RSA is a serious disease involving fetus/baby and mother. Misinformation about RSA may lead to more RSA. Therefore, the results of this study should be interpreted cautiously. Further clinical studies as well as in vitro or in vivo studies are still warranted to investigate the effects of Wenjing decoction on RSA.

\section{Conclusions}

We selected 100 intersected target proteins of the active ingredients by cross-validation. During RSA, the TNF- $\alpha, I L-$ 10, TLR4, JUN, IL-1B, CYBB, PTGS2, APOE, SPI1, and MPO pathways might be targeted by the Wenjing decoction. Furthermore, "C-type lectin receptor signaling pathway," "chemokine signaling pathway," "leukocyte transendothelial migration," "TNF signaling pathway," "MAPK signaling pathway," and "AGE-RAGE signaling pathway in diabetic complications" might correlate with the pharmacological activity of Wenjing decoction. However, the genes and pathways involved and their significance in women with RSA treated by Wenjing decoction should be explored further.

\section{Abbreviation}

RSA: Recurrent spontaneous abortion

DEGs: Differentially expressed genes

PPI: Protein-protein interaction

TNF- $\alpha$ : Tumor necrosis factor- $\alpha$

IL-10: Interleukin-10

TLR4: Toll-like receptor 4

JUN: Jun proto-oncogene

IL-1B: Interleukin-1-beta

CYBB: Cytochrome b-245 beta chain

PTGS2: Prostaglandin-endoperoxide synthase 2

APOE: Apolipoprotein E

SPI1: $\quad$ Salmonella pathogenicity island 1

MPO: $\quad$ Myeloperoxidase

TCM: Traditional Chinese medicine

MW: $\quad$ Molecular weight

Hdon/ The number of hydrogen bond donors/

Hacc: acceptors

BBB: $\quad$ Blood-brain barrier

DL: $\quad$ Drug-likeness

OB: $\quad$ Oral bioavailability
HL: $\quad$ Drug half-life

ADME: Absorption, distribution, metabolism, and elimination

TTD: $\quad$ Therapeutic Target Database

KEGG: Kyoto Encyclopedia of Genes and Genomes

GEO: Gene Expression Omnibus

CPM: $\quad$ Counts per million

FC: $\quad$ Fold change

GO: $\quad$ Gene Ontology

BP: Biological process.

\section{Data Availability}

The data supporting the conclusions of the study could be accessed by reasonable request from the corresponding author.

\section{Additional Points}

(1) In total, 100 intersecting target proteins were selected by the Venn diagram. (2) Enrichment analysis highlighted 24 pathways for the intersecting target proteins. (3) The key targets include TNF- $\alpha, I L-10$, TLR4, JUN, IL-1B, CYBB, PTGS2, APOE, SPI1, and MPO.

\section{Conflicts of Interest}

The authors declare that they have no conflicts of interest.

\section{Supplementary Materials}

The list of the 100 intersection genes. (Supplementary Materials)

\section{References}

[1] M. H. K. Lang, "Abortion: taking the debate seriously," Medwave, vol. 15, no. 4, p. e6139, 2015.

[2] L. Jacob, C. Gerhard, K. Kostev, and M. Kalder, "Association between induced abortion, spontaneous abortion, and infertility respectively and the risk of psychiatric disorders in 57,770 women followed in gynecological practices in Germany," Journal of Affective Disorders, vol. 251, pp. 107-113, 2019.

[3] R. L. Chan, A. F. Olshan, D. A. Savitz et al., "Severity and duration of nausea and vomiting symptoms in pregnancy and spontaneous abortion," Human Reproduction, vol. 25, no. 11, pp. 2907-2912, 2010.

[4] C. J. Cao, Y. F. Wang, D. M. Fang, and Y. Hu, "Relation between mycoplasma infection and recurrent spontaneous abortion," European Review for Medical and Pharmacological Sciences, vol. 22, no. 8, pp. 2207-2211, 2018.

[5] M. H. Pinar, K. Gibbins, M. He, S. Kostadinov, and R. Silver, "Early pregnancy losses: review of nomenclature, histopathology, and possible etiologies," Fetal and Pediatric Pathology, vol. 37, no. 3, pp. 191-209, 2018.

[6] C. B. Polis, C. Mhango, J. Philbin, W. Chimwaza, E. Chipeta, and A. Msusa, "Incidence of induced abortion in Malawi, 2015," PLoS One, vol. 12, no. 4, Article ID e0173639, 2017. 
[7] G.-Y. Yang, H. Luo, X. Liao, and J.-P. Liu, "Chinese herbal medicine for the treatment of recurrent miscarriage: a systematic review of randomized clinical trials," BMC Complementary \& Alternative Medicine, vol. 13, no. 1, p. 320, 2013.

[8] L. Xu, M. Li, H. Zhou et al., "Rapid characterization of the chemical constituents and rat metabolites of the Wen-Jing decoction by ultra high performance liquid chromatography coupled with electrospray ionization quadrupole time-offlight tandem mass spectrometry," Journal of Separation Science, vol. 42, no. 6, pp. 1174-1193, 2019.

[9] L. Gao, C. Jia, H. Zhang, and C. Ma, "Wenjing decoction (herbal medicine) for the treatment of primary dysmenorrhea: a systematic review and meta-analysis," Archives of Gynecology and Obstetrics, vol. 296, no. 4, pp. 679-689, 2017.

[10] D. J. Xu, H. L. Du, and X. M. Cheng, "Effects of modified Wenjing mecoction on the ovarian oxidative damage of cold coagulation blood stasis model rats," Chinese Journal of Integrated Traditional and Western Medicine, vol. 32, no. 1, pp. 58-60, 2012.

[11] D. Wang, X. Cheng, H. Fang et al., "Effects of modified wenjing decoction on microcirculation in reproductive organs in rats with symptom patterns of cold coagulation and blood stasis," Journal of Traditional Chinese Medicine, vol. 40, no. 2, pp. 212-223, 2020.

[12] Y. Ma, K. Zhou, J. Fan, and S. Sun, "Traditional Chinese medicine: potential approaches from modern dynamical complexity theories," Frontiers of Medicine, vol. 10, no. 1, pp. 28-32, 2016.

[13] A. X. Lin, G. Chan, Y. Hu et al., "Internationalization of traditional Chinese medicine: current international market, internationalization challenges and prospective suggestions," Chinese Medicine, vol. 13, no. 1, p. 9, 2018.

[14] J. Ru, P. Li, J. Wang et al., "TCMSP: a database of systems pharmacology for drug discovery from herbal medicines," Journal of Cheminformatics, vol. 6, no. 1, p. 13, 2014.

[15] E. Markovsky, H. Baabur-Cohen, A. Eldar-Boock et al., "distribution, metabolism and elimination of polymer therapeutics," Journal of Controlled Release Official Journal of the Controlled Release Society, vol. 161, no. 2, 2012.

[16] Z. Zhuang, Q. Chen, C. Huang, J. Wen, H. Huang, and Z. Liu, "A comprehensive network pharmacology-based strategy to investigate multiple mechanisms of HeChan tablet on lung cancer," Evidence-based Complementary and Alternative Medicine, vol. 2020, Article ID 7658342, 17 pages, 2020.

[17] Z. Huang, G. Du, X. Huang et al., "The enhancer RNA lncSLC4A1-1 epigenetically regulates unexplained recurrent pregnancy loss (URPL) by activating CXCL8 and NF-kB pathway," EBioMedicine, vol. 38, pp. 162-170, 2018.

[18] S. Ghosh and C.-K. K. Chan, "Analysis of RNA-seq data using TopHat and cufflinks," Plant Bioinformatics, vol. 1374, p. 339, 2016.

[19] L. Yang, G. K. Smyth, and S. Wei, "FeatureCounts: an efficient general purpose program for assigning sequence reads to genomic features," Bioinformatics, vol. 30, no. 7, pp. 923-930, 2014.

[20] J. C. Wright, J. Mudge, H. Weisser et al., "Improving GENCODE reference gene annotation using a high-stringency proteogenomics workflow," Nature Communications, vol. 7, no. 1, p. 11778, 2016.

[21] M. D. Robinson, D. J. Mccarthy, and G. K. Smyth, “edgeR: a bioconductor package for differential expression analysis of digital gene expression data," Bioinformatics, vol. 26, no. 1, pp. 139-140, 2010.
[22] J. H. Li, J. N. Law, and T. M. Murali, "Automating the PathLinker app for cytoscape," F1000research, vol. 7, p. 727, 2018.

[23] G. Yu, L.-G. Wang, Y. Han, and Q.-Y. He, “Cluster profiler: an $\mathrm{R}$ Package for comparing biological themes among gene clusters," OMICS: A Journal of Integrative Biology, vol. 16, no. 5, pp. 284-287, 2012.

[24] D. Szklarczyk, A. L. Gable, D. Lyon et al., "STRING v11: protein-protein association networks with increased coverage, supporting functional discovery in genome-wide experimental datasets," Nucleic Acids Research, vol. 47, no. 1, pp. D607-D613, 2019.

[25] L. Song, W. Lei, Z. Xing, Y. Huang, and Z. Miao, "Expression level of TNF- $\alpha$ in decidual tissue and peripheral blood of patients with recurrent spontaneous abortion," Central-European Journal of Immunology, vol. 2, pp. 156-160, 2017.

[26] C. Zhang, X. Deng, X. Zhang et al., "Association between serum TNF- $\alpha$ levels and recurrent spontaneous miscarriage: a meta-analysis," American Journal of Reproductive Immunology, vol. 75, no. 2, pp. 86-93, 2016.

[27] F. Y. Azizieh and R. Raghupathy, "IL-10 and pregnancy complications," Clinical and Experimental Obstetrics \& Gynecology, vol. 44, no. 2, pp. 252-258, 2017.

[28] P. Li, H. L. Wu, and B. H. Dong, "Relationship between TLR4 and CCL2 expression and recurrent spontaneous abortion," Genetics and Molecular Research, vol. 15, no. 1, p. 6882, 2016.

[29] N. Sereshki, A. Andalib, A. Ghahiri, F. Mehrabian, R. Sherkat, and A. Rezaei, "Decreased toll-like receptor (TLR) 2 and 4 expression in spermatozoa in couples with unexplained recurrent spontaneous abortion (URSA)," Iranian Journal of Allergy, Asthma, and Immunology, vol. 18, no. 6, pp. 701-706, 2019.

[30] J. Ma, X. Zhang, G. He, and C. Yang, "Association between TNF, IL1B, IL6, IL10 and IFNG polymorphisms and recurrent miscarriage: a case control study," Reproductive Biology \& Endocrinology, vol. 15, no. 1, p. 83, 2017.

[31] S. J. Yue, L. T. Xin, Y. C. Fan et al., "Herb pair DangguiHonghua: mechanisms underlying blood stasis syndrome by system pharmacology approach," Scientific Reports, vol. 7, no. 1, p. 40318, 2017.

[32] C. Goodman, C. S. Goodman, J. Hur, R. S. Jeyendran, and C. Coulam, "The association of apoprotein E polymorphisms with recurrent pregnancy loss," American Journal of Reproductive Immunology, vol. 61, no. 1, pp. 34-38, 2010.

[33] R. Azizi, M. S. Soltani-Zangbar, G. Sheikhansari et al., "Metabolic syndrome mediates inflammatory and oxidative stress responses in patients with recurrent pregnancy loss," Journal of Reproductive Immunology, vol. 133, pp. 18-26, 2019.

[34] B. A. Wevers, T. B. Geijtenbeek, and S. I. Gringhuis, "C-type lectin receptors orchestrate antifungal immunity," Future Microbiology, vol. 8, no. 7, pp. 839-854, 2013.

[35] Q.-D. Lin and L.-H. Qiu, "Pathogenesis, diagnosis, and treatment of recurrent spontaneous abortion with immune type," Frontiers of Medicine in China, vol. 4, no. 3, pp. 275-279, 2010.

[36] B. Van Iddekinge, G. J. Hofmeyr, W. R. Bezwoda, A. A. Wadee, and P. Van Rooy, "Recurrent spontaneous abortion: histocompatibility between partners, response to immune therapy, and subsequent reproductive performance," American Journal of Reproductive Immunology, vol. 30, no. 1, pp. 37-44, 2013.

[37] M. Mellado, J. M. Rodríguez-Frade, S. Mañes, and C. Martínez-A, "Chemokine signaling and functional responses: the role of receptor dimerization and TK pathway 
activation," Annual Review of Immunology, vol. 19, no. 1, pp. 397-421, 2001.

[38] S. Nourshargh and R. Alon, "Leukocyte migration into inflamed tissues," Immunity, vol. 41, no. 5, pp. 694-707, 2014.

[39] M. Baumann, "Role of advanced glycation end products in hypertension and cardiovascular risk: human studies," Journal of the American Society of Hypertension, vol. 6, no. 6, pp. 427-435, 2012. 